\title{
A STUDY ON PRESENTATION AND BEHAVIOR OF COLO-RECTAL CARCINOMA
} IN JHARKHAND

\section{Dr. Navin Kumar Saha \\ Prof. (Dr.) Mritunjay Sarawgi*}

\section{Dr.C.P.Sinha}

\section{Dr. Krishan Kumar}

Post-graduate student, Dept of General Surgery, RIMS, Ranchi, Jharkhand834009.

MS, Professor, Dept of General Surgery, RIMS, Ranchi, Jharkhand834009. * Corresponding Author

MS,MCH, Associate professor, Dept of General Surgery, RIMS, Ranchi, Jharkhand-834009.

Post-graduate student, Dept of General Surgery, RIMS, Ranchi, Jharkhand834009.

ABSTRACT Introduction: Colo-rectal carcinoma is most frequent neoplasm of Western countries. It is 3rd most common in men and 2nd most common in women. The Aim of this study is to know different presentations and age variations and its method of diagnosis and management. Materials and methods: This is a observational study including 25 patients presented in the Emergency and OPD of RIMS, Ranchi with complaints of bleeding PR, alteration of bowel habits, rectal pain and discharge, incontinence, abdominal pain and distention and loss of weight. Results: Most common age of presentation is 40-50 years, most commonly involving men. Most common presentation is alteration of bowel habits followed by bleeding PR. Diagnosis was mostly made by CECT whole abdomen and colonoscopic /Rectal biopsy. Follow-up was done by CEA levels. Conclusion: There is increased incidence of colo-rectal carcinoma in young patients and there is tendency of late presentation of colo-rectal cancers.

\section{KEYWORDS : Colo-rectal cancer, altered bowel habit, Bleeding per rectum, colonoscopy}

\section{INTRODUCTION:}

Colorectal cancer (CRC) is a formidable health problem worldwide. In the world colorectal cancer is the second most common cancer in women after breast cancer and the third most common in men after lung and prostate cancer $(1,2)$.

In India, the annual incidence rates (AARs) for colon cancer and rectal cancer in men are 4.4 and 4.1 per 100000 , respectively. The AAR for colon cancer in women is 3.9 per 100000. Colon cancer is the 8th most common and rectal cancer 9th most common among men. For women, rectal cancer is not included in the top 10 common cancers, whereas colon cancer is 9 th most common cancer (3).

In fact, $5.7 \%$ of patients with newly diagnosed colorectal cancer are under 45 years of age and $20.5 \%$ are younger than 55 years of age [1]. For individuals under age 50 , the incidence of colorectal cancer rose by $22 \%$ from 2000 to 2013 , mostly accounted for by tumours of the distal colon and rectum (4). Incidence strongly varies globally and is closely linked to elements of a so-called western lifestyle. Incidence is higher in men than women and strongly increases with age (5).

Recent UK data reports a delay of 10 months between onset of symptoms and treatment of CRC, with a median patient delay of 3 months, usually because the patient does not think the symptoms are serious. CRC contributes to significant morbidity, and mortality rates are reported to be on the increase in low-resource settings (6). However CRC has good prognosis when managed well in early stages, with a 5-year survival rate of up to $90 \%$ (7).

\section{AIMS AND OBJECTIVES:}

The aim of this study is to know different presentations and age variations of colorectal carcinoma patients and its method of diagnosis and management. To reduce the the mortality and morbidity of the disease by diagnosing it in early stage.

To diagnose the colorectal cancer in the early stages, so that radical care of disease as early as possible.

\section{MATERIAL AND METHODS:}

Source of data: Patients of colorectal carcinoma admitted in Department of General surgery coming to RIMS, Ranchi.

Type of study: observational study.

Period of study: One and half year (Jan2019 to June 2020)

Place of study: Indoor patients in the Department of Surgery at RIMS, Ranchi.

Sample size: 25 cases of colorectal carcinoma.

Plan for analysing data : Patients came with complains of bleeding per rectum, alteration in bowel habit, rectal pain and rectal discharge, abdominal pain and abdominal distension, incontinence, loss of weight and Features of intestinal obstruction and others non-specific symptoms. All general examination ,per abdominal examination and digital rectal examination was done on every patient's followed by colonoscopy and colonoscopic/rectal biopsy followed by CECT of whole abdomen. Some patients presented with acute intestinal obstruction were operated and colonic mass was found. Preoperative and postoperative serum CEA was done.

\section{RESULT:}

In the present study a total 25 cases were included having age groups of $20-70$ years out of which $15(60 \%)$ were male and 10 (40\%) were female amounting to a male-female ratio of approximately $1.5: 1$.

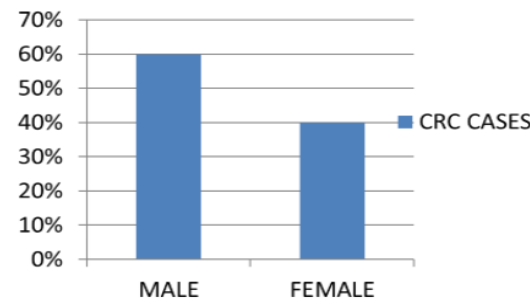

On the basis of age, below 40 years there were 6, between 40 50years 9 patients,51-60years 6 patients and above 60years only 4 patients. 


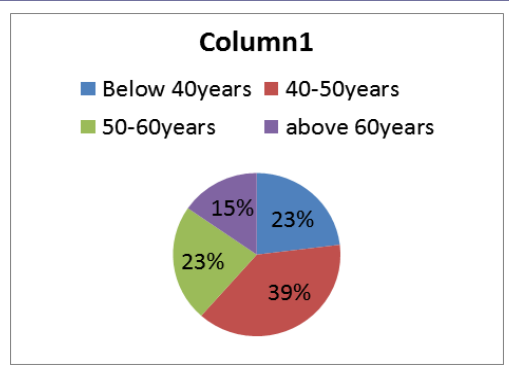

On the basis of site of colorectal cancer,
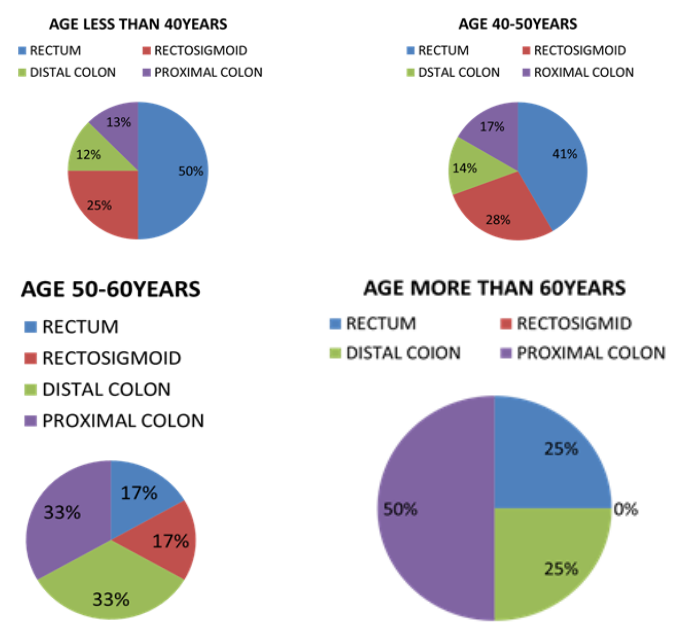

Out of 25 patients ,8 (32\%) patients are presented with the feature of either acute intestinal obstruction or subacute intestinal obstruction.

Most common presentation in CRC is alteration of bowel habit in $15(60 \%)$ patients followed by bleeding per rectum in 12 (around $50 \%$ ) patients.

\section{DISCUSSION:}

A number of environmental and genetic risk factors have been identified for CRC these include- Age, Nutrition, Low physical activity, Inflammatory bowel disease, Genetic factors

It is estimated that about $80 \%$ of all cases of CRC are caused by diet alone. However, heritable factors account for $35 \%$ of the risk of developing CRC. These heritable factors can be considered in two groups:

- High-penetration autosomal dominant syndromes familial adenomatous polyposis (FAP and variants) and hereditary non-polyposis colorectal cancer (HNPCC), which represent $2 \%-5 \%$ of all colorectal cancers and is associated with an $80 \%$ lifetime risk.

- Familial clustering is likely to have a multifactorial mode of inheritance. Several genes are likely to be involved, some may predispose to adenomatous polyp formation. The mode of inheritance is autosomal dominant but with $a$ low penetrance. The key determinants of risk are the youngest age of onset of CRC and the number of firstdegree relatives involved.

Proximal disease are presented with iron deficiency anemia (microcytic, hypochromic), an abdominal mass, or as an emergency presentation with signs and symptoms of intestinal obstruction.

However, for left colon and rectal cancer, the presentation is usually with rectal bleeding and a change in bowel habit, which is usually an increased frequency of defecation and/or looser stools.
Rectal bleeding occurs without anal symptoms in over $60 \%$ of patients. In very low rectal cancer, the symptom of tenesmus the feeling of incomplete evacuation - may occur, and anal pain usually indicates that invasion of the anal sphincter has occurred.

Colonoscopy is the current reference method for CRC screening and is recommended every 10 years in average-risk patients aged 50 years or older. The ability of colonoscopy to detect both cancerous and precancerous lesions via direct visualization has been demonstrated in several large cohort studies. The sensitivity of colonoscopy for detecting CRC is $>95 \%$, while its sensitivity for detecting advanced adenomas $(\geq 10 \mathrm{~mm}$ in diameter) is $88 \%-98$.

\section{CONCLUSION:}

There was slight male predominance in colorectal carcinoma patients in Jharkhand with most common age was $40-50$ years (around 40\%).

There is increasing incidence of colorectal carcinoma in young adult of age less than 40years and more common distal colon or primary rectal cancer in comparative to old age patients.

$32 \%$ of total patients presented late after the feature of intestinal obstruction lead to increase in mortality and morbidity of patients. CRC has good prognosis when managed well in early stages.

To conclude, since CRC poses a significant burden on the community in our setting due to its late presentation, its early diagnosis and treatment is necessary to reduce morbidity and mortality which can be done through effective screening and educational campaigns. These must be coupled with research into effective and age-appropriate specific screening methods for our setting, together with additional research exploring molecular etiologies of these cancers and their link to infections prevalent in the region.

\section{REFERENCES:}

1. Boyle P, Ferlay J, Cancer incidence and mortality in Europe 2004. Ann Oncol 2005;16:481-8.

2. Office for National Statistics. Cancer statistics registrations: registrations of cancer diagnosed in 2004, England.

3. NCRP (2013) Three-year report of the population based cancer registries2009-2011. National cancer registry programme, Indian council of medical research (ICMR), Bangalore, India, 2013

4. Siegel RL, Miller KD, Fedewa SA, et al. Colorectal cancer statistics, 2017. CA Cancer J Clin. 2017 Mar 1. [Epub ahead of print]

5. Brenner H, Kloor M, Pox CP. Colorectal cancer. Lancet. 2014 Apr 26;383(9927):1490-1502. doi: 10.1016/S0140-6736(13)61649-9. Epub 2013 Nov 11. PMID: 24225001.

6. Center MM, Jemal A, Smith RA, et al. Worldwide variations in colorectal cancer. Dis Colon Rectum. 2010;53:1099.

7. Center MM, Jemal A, Smith RA, et al. Worldwide variations in colorectal cancer. Dis Colon Rectum. 2010;53:1099. 